\title{
Parental involvement does not augment the effectiveness of an intense behavioral program for the treatment of childhood obesity
}

\author{
Eirini Bathrellou' ${ }^{1}$, Mary Yannakoulia ${ }^{1}$, Katerina Papanikolaou ${ }^{2}$, Artemios Pehlivanidis ${ }^{3}$, \\ Panagiota Pervanidou ${ }^{4}$, Christina Kanaka-Gantenbein ${ }^{4}$, Ioanna Tokou ${ }^{1}$, John Tsiantis ${ }^{2}$, \\ George P. Chrousos ${ }^{4}$, Labros S. Sidossis ${ }^{1}$
}

\begin{abstract}
${ }^{1}$ Department of Nutrition and Dietetics, Harokopio University, ${ }^{2}$ Department of Child Psychiatry, University of Athens Medical School, "Agia Sophia" Children's Hospital, ${ }^{3}$ Department of Psychiatry, Cognitive Therapy Unit, University of Athens Medical School, Eginition Hospital, ${ }^{4}$ Unit of Endocrinology, Metabolism and Diabetes, First Department of Pediatrics, University of Athens Medical School, “Agia Sophia” Children's Hospital, Athens, Greece
\end{abstract}

\begin{abstract}
OBJECTIVE: To evaluate the effectiveness of active parental involvement in a lifestyle intervention for the management of childhood obesity. DESIGN: Forty-two overweight children (32 girls and 10 boys), aged $9.2 \pm 0.2$ years and with percent overweight $39.8 \pm 2.7 \%$, were randomly allocated either to a child-and-parent group $(N=23)$ or a child-alone group $(N=19)$. Both groups attended a 3-month multidisciplinary program extended by booster sessions during follow-up, which involved many cognitive behavioral therapy principles and assigned high self-regulation to the children, but differed in parental involvement. Percent overweight was evaluated at baseline, and at 3, 6, and 18 months thereafter. RESULTS: There was no significant interaction between time and group or a significant difference between groups. Percent overweight decreased by $4.9 \pm 1.4$ at 18 months $(p<0.001)$; the reduction occurred during the active phase of the treatment (0-3 months) and was maintained thereafter. CONCLUSION: In the setting of the present study, the active parental involvement did not significantly modify the results of lifestyle interventions for children's overweight management.
\end{abstract}

Key words: Children, Cognitive behavioral therapy, Obesity, Parents, Weight management

Address for correspondence:

Labros S. Sidossis, PhD, Professor of Nutrition and Dietetics,

Department of Nutrition and Dietetics, Harokopio University,

70 E. Venizelou Ave, 17671 Athens, Greece,

Tel.: +30210 9549 154, Fax: +30210 9549 141,

e-mail: lsidossis@hua.gr

Received 03-08-09, Revised 05-12-09, Accepted 10-02-10

\section{INTRODUCTION}

Recommendations for treating childhood obesity focus on lifestyle modification in terms of dietary and physical activity intervention applied through behavioral modification techniques., ${ }^{1,2}$ Parental support is a key component of most interventions. ${ }^{3,4}$ Parents 
affect children's eating and physical activity patterns by several means, namely formulating children's environment, being role models, and controlling their dietary intake, ${ }^{5}$ and are thus incorporated in a wide range of dietary interventions targeting children. ${ }^{6,7}$ Regarding obesity management, parents have been assigned different roles. Epstein et al strongly supported the role of parents as targets for managing their own weight along with their child's effort, ${ }^{8}$ while Golan et al proposed that parents should be the exclusive agents of change without any direct child involvement. ${ }^{9} \mathrm{On}$ the other hand, Israel et al found that training children in self-regulatory techniques, such as self-monitoring, self-assessment, and self-consequencing, compared to assigning parents most responsibility for change, was a more successful approach for maintaining percent overweight loss after treatment. ${ }^{10}$ As data are not conclusive ${ }^{11}$ re-evaluation of parental participation in the management of children's overweight is needed, especially since children's eating habits and behaviors have undergone great changes over the past few decates. Children have many opportunities to eat without parental supervision and can easily access foods and beverages in their social environment. ${ }^{12,13}$ One may therefore argue that parental involvement these days may be a component of lesser importance within a childhood obesity program. In this context, the aim of the present study was to evaluate the effectiveness of involving parents in an intense childhood obesity program involving lifestyle intervention based on cognitive behavioral therapy (CBT) principles and assigning high self-management to the children.

\section{METHODS}

Overweight or obese children, aged between 7 and 12 years, without any chronic physical or mental illness, were recruited from the "Agia Sofia" Children's Hospital, a major public pediatric hospital in Athens, Greece. Approval to conduct the study was obtained from both the Ethics Committee of Harokopio University and the Ethics Committee of "Agia Sophia" Children's Hospital, and all parents provided their written consent. Forty-seven children were randomly allocated to one of two study groups: (i) Child-and-parent group ( $\mathrm{N}=24)$ and (ii) Child- alone group $(\mathrm{N}=23)$.

The intervention has been described in detail elsewhere. ${ }^{14}$ Briefly, it was a multidisciplinary program involving many CBT principles and some novelties as regards the dietetic practice, such as supervision of the dieticians by the psychiatrists and implementation with booster sessions after the intensive program (6 monthly sessions from 3 to 9 months and one final session at 5 months). The intensive program consisted of 12 weekly sessions, each lasting 1 hour and conducted individually. In the Child-and-parent group, parents were asked to act as helpers: apart from attending two individual sessions with the dietician, they also participated in the last 10 minutes of each session, while their cooperation was actively requested in supporting their child to implement the goals set. In the Child-alone group, sessions were conducted without any parental involvement and parental help was not required unless the child requested it.

Body weight and height were measured and Body Mass Index (BMI) was calculated at baseline and at 3, 6 , and 18 months after the initiation of the program. The main outcome measure was percent overweight, calculated as [(current BMI - BMI cut-off for overweight)/ BMI cut-off for overweight] x 100, where BMI cut-offs were the age- and sex-specific BMI cut-off points for children and adolescents, which correspond to the adult BMI cut-off point for overweight adopted by the International Obesity Task Force. ${ }^{15}$

The effect of group (Child-and-parent vs. Childalone) and time ( 0 vs. 3 vs. 6 vs. 18 months) was tested by using repeated measures analysis of variance (ANOVA) for fixed effects. Univariate tests were interpreted based on whether or not the assumption of sphericity (Mauchly's W test) was met. Differences between time points were evaluated by applying appropriate statistical contrasts. Differences between groups at baseline were assessed by using Student's independent t-test. Normality was tested by the Kolmogorov-Smirnov one-sample test and homogeneity by Levene's tests. Continuous variables are presented as mean \pm SEM. A $p$-value $<0.05$ was considered statistically significant. All statistical analyses were performed with statistical software SPSS 17.0 (SPSS Inc., Chicago, IL). 


\section{RESULTS}

Of the 47 children who were originally allocated to the two study groups, five never attended the program, so that 42 children participated in the baseline assessment: 23 in the Child-and-parent group and 19 in the Child-alone group. Their characteristics are presented in Table 1 . Five children (4 in the Child-and-parent and 1 in the Child-alone group) dropped out before completing half of the intervention. At the 6- and 18-month assessment, 36 and 32 children, respectively, were available for measurement (18 and 16 children in each group, at each time point). Children who were available for measurement at the 3- and 6-month assessment did not differ in their baseline characteristics compared to those who were not; however, those available for the 18-month assessment were shorter $(p=0.048)$ and tended to have lower body weight $(p=0.076)$, but did not differ in BMI $(p=0.298)$ and percent overweight $(p=0.562)$.

For the children who were available for measurements at all time points of assessment, a significant effect of time in percent overweight was found ( $p$ $<0.001$ ), which was the same for both groups ( $\mathrm{p}$ for time by group interaction $=0.670, p$ for group differences $=0.311)$. The percent overweight significantly decreased from baseline to 3 months (from 38.9 \pm 3.2 to $35.5 \pm 3.1$, respectively; $p<0.001$ ), but did not change thereafter $(p>0.380)$ (Figure 1). At 18 months, it was $33.9 \pm 3.5$, being $4.9 \pm 1.4$ lower than baseline $(p$ $=0.002)$.

\section{DISCUSSION}

We assessed the effectiveness of parental involvement in an intense multidisciplinary lifestyle modi-

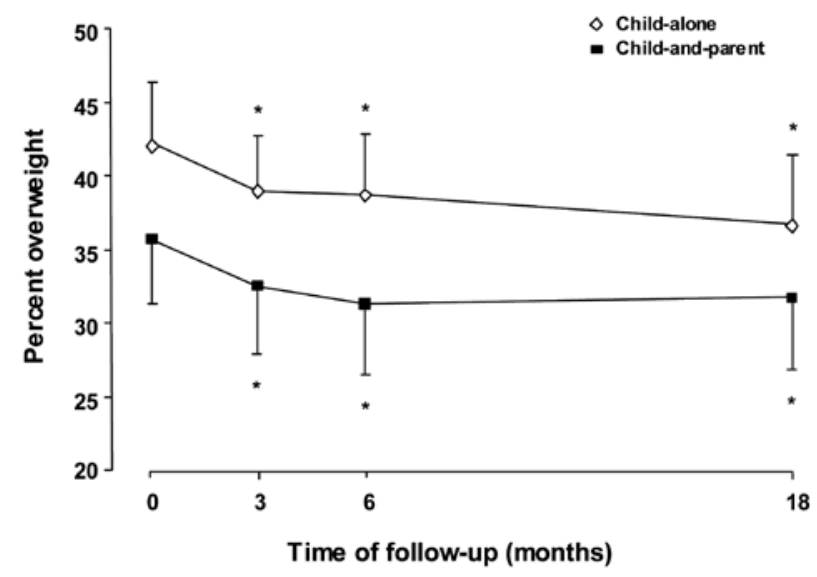

Figure 1. Percent overweight in the two study groups at 3,6, and 18 months of follow-up ( $\mathrm{N}=32,16$ in each group). Percent overweight decreased during the active phase of the treatment (0-3 months) and was maintained thereafter in both groups. Values are means \pm SEM.* Value different from baseline within each group, $p<0.05$.

fication program for the management of childhood obesity, assigning high self-regulation responsibilities to the children. We found no additional benefit when parental help was actively requested during the program. The role of parents has attracted much attention with respect to their children's overweight management, as parents can affect many aspects of the children's dietary and physical activity environment. ${ }^{16}$ While increased parental participation has long been proven to be effective, ${ }^{17}$ there are also findings in favor of an enhanced child involvement approach..$^{10}$ Training children in self-regulatory skills regarding their food and physical activity habits may be of great importance, considering that nowadays children have many opportunities throughout the day to choose their own food. ${ }^{12,13}$. Our results indicate that such an approach is as effective as if active parental

Table 1. Subjects' characteristics at baseline $(\mathrm{N}=42)$.

\begin{tabular}{lccc}
\hline & Child-alone & Child-and-parent & $p$-value \\
\hline $\mathrm{N}$ & 19 & 23 & \\
Age $(\mathrm{y})$ & $9.1 \pm 0.3$ & $9.4 \pm 0.3$ & 0.527 \\
Height $(\mathrm{cm})$ & $139 \pm 2$ & $140 \pm 2$ & 0.805 \\
Weight $(\mathrm{kg})$ & $53.3 \pm 2.8$ & $52.4 \pm 2.3$ & 0.802 \\
Body Mass Index $\left(\mathrm{kg} / \mathrm{m}^{2}\right)$ & $27.4 \pm 0.7$ & $26.7 \pm 0.8$ & 0.528 \\
Percent overweight & $42.5 \pm 3.9$ & $37.5 \pm 3.8$ & 0.367 \\
\hline
\end{tabular}

Values are means \pm SEM. $p$-values from Student's independent t-test. 
involvement was included. However, the finding may apply to the particular degree of parental involvement and the timing of follow-up assessed; thus, it might be possible that a beneficial effect in favor of the Childand-parent group could be obtained by a higher degree of parental participation or after a longer follow-up period. Moreover, we cannot exclude the possibility that parents had already been involved in their child's body weight regulation practices, so that the current active involvement did not add any benefit.

The percent overweight in the present study decreased by approximately 5 percentage units. This is a rather moderate effect as compared with the results of previous studies. ${ }^{17,18}$ It must be mentioned however that data on the clinical significance of this change are lacking, namely what degree of reduction in percent overweight produces favorable metabolic alterations in the pediatric population. Moreover, even though favorable changes produced by similar interventions fade away as time of follow-up increases, ${ }^{19,20}$ percent overweight loss in our study was maintained throughout follow-up. This is a strong point of our intervention and could perhaps be attributed to the implementation of booster sessions, extending time and contact of intervention. However, the decrease in percent overweight was achieved during the active phase of intervention, implying the need for continuing intervention at follow-up, as is also indicated in the current literature. ${ }^{21}$

Our study has several limitations. Although most children attended the intensive phase of the intervention (88\%), only three quarters of the children completed all stages of the 18-month follow-up assessment. Our attrition rate, however, is similar to that previously achieved in studies related to the treatment of childhood obesity. ${ }^{17}$ Though the lack of a control group could be considered as a limitation, one should bear in mind that non-intervention groups of overweight children are difficult to obtain. After all, as children are left untreated, they usually increase their overweight status over time, ${ }^{20}$ thus the inclusion of such a group would possibly increase the effectiveness of our intervention.

In conclusion, in our study parental involvement did not augment the effectiveness of an intense lifestyle intervention for the management of childhood obesity assigning high self-regulation to the children, either in the short or in the long term. More research is obviously needed to confirm these findings in larger pediatric samples studied for a longer period.

\section{ACKNOWLEDGEMENTS}

No conflict of interest to declare. The study was funded in part by the Department of Nutrition and Dietetics Graduate Program.

\section{REFERENCES}

1. August GP, Caprio S, Fennoy I, et al, 2008 Prevention and treatment of pediatric obesity: an endocrine society clinical practice guideline based on expert opinion. $\mathbf{J}$ Clin Endocrinol Metab 93: 4576-4599.

2. Spear BA, Barlow SE, Ervin C, et al, 2007 Recommendations for treatment of child and adolescent overweight and obesity. Pediatrics 120: S254-288.

3. Barlow SE, 2007 Expert committee recommendations regarding the prevention, assessment, and treatment of child and adolescent overweight and obesity: summary report. Pediatrics 120: Suppl 4: 164-192.

4. Gilles A, Cassano M, Shepherd EJ, Higgins D, Hecker JE, Nangle DW, 2008 Comparing active pediatric obesity treatments using meta-analysis. J Clin Child Adolesc Psychol 37: 886-892.

5. Johnson-Taylor WL, Everhart JE, 2006 Modifiable environmental and behavioral determinants of overweight among children and adolescents: report of a workshop. Obesity (Silver Spring) 14: 929-966.

6. Perry CL, Luepker RV, Murray DM, et al, 1988 Parent involvement with children's health promotion: the Minnesota Home Team. Am J Public Health 78: 11561160 .

7. O’Dougherty M, Story M, Stang J, 2006 Observations of parent-child co-shoppers in supermarkets: children's involvement in food selections, parental yielding, and refusal strategies. J Nutr Educ Behav 38: 183-188.

8. Epstein LH, Valoski A, Wing RR, McCurley J, 1990 Ten-year follow-up of behavioral, family-based treatment for obese children. JAMA 264: 2519-2523.

9. Golan M, Weizman A, Apter A, Fainaru M, 1998 Parents as the exclusive agents of change in the treatment of childhood obesity. Am J Clin Nutr 67: 1130-1135.

10. Israel AC, Guile CA, Baker JE, Silverman WK, 1994 An evaluation of enhanced self-regulation training in the treatment of childhood obesity. J Pediatr Psychol 19: 737-749.

11. McGovern L, Johnson JN, Paulo R, et al, 2008 Clinical review: treatment of pediatric obesity: a systematic review and meta-analysis of randomized trials. J Clin Endocrinol Metab 93: 4600-4605. 
12. Briefel RR, Wilson A, Gleason PM, 2009 Consumption of low-nutrient, energy-dense foods and beverages at school, home, and other locations among school lunch participants and nonparticipants. J Am Diet Assoc 109: S79-90.

13. Hawkins SS, Cole TJ, Law C, 2009 Examining the relationship between maternal employment and health behaviours in 5-year-old British children. J Epidemiol Community Health 63: 994-1004.

14. Bathrellou E, Yannakoulia M, Papanikolaou K, et al, 2010 Development of a multidisciplinary intervention for the treatment of childhood obesity based on Cognitive Behavioral Therapy. Child \& Family Behavior Therapy 32: 34-50.

15. Cole TJ, Bellizzi MC, Flegal KM, Dietz WH 2000, Establishing a standard definition for child overweight and obesity worldwide: international survey. BMJ 320: 1240-1243.

16. Birch LL, Fisher JO, 1998 Development of eating behaviors among children and adolescents. Pediatrics
101: 539-549.

17. Oude Luttikhuis H, Baur L, Jansen H, et al, 2009 Interventions for treating obesity in children. Cochrane Database Syst Rev: CD001872.

18. Jelalian E, Saelens BE, 1999 Empirically supported treatments in pediatric psychology: pediatric obesity. J Pediatr Psychol 24: 223-248.

19. Epstein LH, Paluch RA, Roemmich JN, Beecher MD, 2007 Family-based obesity treatment, then and now: twenty-five years of pediatric obesity treatment. Health Psychol 26: 381-391.

20. Wilfley DE, Tibbs TL, Van Buren DJ, Reach KP, Walker MS, Epstein LH, 2007 Lifestyle interventions in the treatment of childhood overweight: a meta-analytic review of randomized controlled trials. Health Psychol 26: 521-532.

21. Wilfley DE, Stein RI, Saelens BE, et al, 2007 Efficacy of maintenance treatment approaches for childhood overweight: a randomized controlled trial. JAMA 298: 1661-1673. 